Proyecciones

Vol. $11 \mathrm{~N}^{\circ} 1$, pp.29-35 Mayo 1992

Universidad Católica del Norte

Antofagasta - Chile

\title{
ALGUNAS $T$-ALGEBRAS DE RANGO 3 CON FORMAS BILINEALES ASOCIATIVAS *
}

\author{
R. BAEZA-VEGA y R. BENAVIDES
}

Unıversidad de la Frontera, Temuco, Chile.

\section{Resumen}

Sean $(\mathbf{A}, \omega)$ una $\mathcal{T}$-álgebra con idempotente, cuya ecuación rango es $x^{3}-$ $(1+\gamma) \omega(x) x^{2}+\gamma \omega(x)^{2} x=0$ y $\mathcal{B}$ una forma bilineal asociativa en $\mathbf{A}$. En este trabajo establecemos que si $0 \neq \gamma \neq 1, B$ es degenerada. Además se demuestra que la simetría de $\mathcal{B}$ sólo depende de la simetría de $\left.\mathcal{B}\right|_{V}$ y que $\mathcal{B}(\mathrm{e}, \mathrm{e})$ es constante para todo idempotente $\mathbf{e} \in \mathbf{A}$.

* Proyectos FONDECYT 165-91 y DIUFRO 9107. 


\section{T-ALGEBRAS.}

En lo que sigue, $K$ es un cuerpo conmutativo de característica diferente de 2 y toda $K$-álgebra es conmutativa, de dimensión finita y no necesariamente asociativa.

Decimos que $(\mathbf{A}, \omega)$ es una $K$-álgebra ponderada si existe un homomorfismo no nulo de álgebras $\omega . \mathbf{A} \rightarrow K \quad$ La $K$-álgebra ponderada $(\mathbf{A}, \omega)$ es una train algebra o simplemente $\mathcal{T}$-álgebra sı existen $r \in \mathbb{N}$ y constantes $\gamma_{1}, \quad, \gamma_{r-1} \in K$. tales que todo $x \in \mathbf{A}$ satisface la igualdad $x^{r}+\gamma_{1} \omega(x) x^{r-1}+\quad+\gamma_{r-1} \omega(x)^{r-1} x=$ 0 El menor de tales $r$ es llamado el rango de A y la ecuación correspondiente es llamada ecuación rango de $\mathbf{A}$

Las siguientes propiedades son válidas en toda $\mathcal{T}$-álgebra $(\mathbf{A}, \omega)$

(a) $1+\gamma_{1}+\quad+\gamma_{r-1}=0$

(b) El núcleo $N$ de $\omega$ es un ideal de codimensión 1 satisfaciendo la identidad $x^{r}=0$

(c) $\omega$ es el único homomorfismo no nulo de $\mathbf{A}$ en $K$

Lema 1.1. Si en la $\mathcal{T}$-álgebra $(\mathbf{A}, \omega)$ vale $\omega(x)\left(x^{2}-\omega(x) x\right)=0$ para todo $x \in \mathbf{A}$. entonces $x^{2}-\omega(x) x=0, \forall x \in \mathbf{A}$ Es decir $(\mathbf{A}, \omega)$ es una $\mathcal{T}$-álgebra de rango 2

Demostración: Notemos que para cualquier elemento $x \notin$ ker $\omega$ vale $x^{2}-$ $\omega(x) x=0 \quad$ Si $x \in$ ker $\omega$ con $x^{2}-\omega(x) x \neq 0$, entonces $x^{2} \neq 0$ pero para cualquier $y \notin \operatorname{ker} \omega$ tenemos $\omega(x+\lambda y)\left((x+\lambda y)^{2}-\omega(x+\lambda y)(x+\lambda y)\right)=0 \forall \lambda \epsilon$ $K$ Luego, en particular $\omega(y) x^{2}=0$, pero $\omega(y) \neq 0$. entonces $x^{2}=0$. Esto indica que necesariamente $x^{2}-\omega(x) x=0 \forall x \in \mathbf{A}$

Proposición 1.2. Sea $(\mathbf{A}, \omega)$ una $\mathcal{T}$-álgebracuya ecuación rango es $x^{3}-(1+$ $\gamma) \omega(x) x^{2}+\gamma \omega(x)^{2} x=0$, entonces $\mathbf{A}$ es una álgebra de Jordan si y sólo si $\gamma=0$ o $\gamma=1$

\section{Demostración:}

$\Leftrightarrow$ Si $\gamma=0 \circ \gamma=1$, la ecuación rango de $\mathbf{A}$ es $x^{3}-\omega(x) x^{2}=0$ o $x^{3}-2 \omega(x) x^{2} x=0$, luego $\mathbf{A}$ es de Jordan por Teorema 2.3.2 [3].

$\Rightarrow$ ) Si A es una álgebra de Jordan, por Teorema 2.3.2 [3], se tiene que la ecuación rango de $\mathbf{A}$ está dada por $x^{3}-\omega(x) x^{2}=0 \circ x^{3}-2 \omega(x) x^{2}+\omega(x)^{2} x=0$

Supongamos que la ecuación es la primera de éstas, restándola de $x^{3}-(1+$ $\gamma) \omega(x) x^{2}+\gamma \omega(x)^{2} x=0$ obtenemos la igualdad $\gamma\left(\omega(x) x^{2}-\omega(x)^{2} x\right)=0$. Si $\gamma \neq 0$, entonces $\omega(x)\left(x^{2}-\omega(x) x\right)=0 \forall x \in \mathbf{A}$, y por el Lema 1.1 anterior 
tenemos que $\mathbf{A}$ es una $\mathcal{T}$-álgebrade rango 2. Esto contradice la hipótesis. luego $\gamma=0$

Análogamente se prueba que si la ecuación rango de $\mathbf{A}$ es $x^{3}-2 \omega(x) x^{2}+$ $\omega(x)^{2} x=0$ entonces $\gamma=1$

Las $T$-álgebras tienen su origen al modelar problemas de tipo genétıco y ahí es frecuente la existencia de un elemento idempotente Por tal razón. consideraremos, de aqui en adelante que la $\mathcal{T}$-álgebra $(\mathbf{A}, \omega)$ tiene un idempotente $\mathbf{e}$ de peso 1 Además sólo consideraremos las $\mathcal{T}$-álgebrasde rango 3 . es decir aquellas que satisfacen la ecuación rango

$$
x^{3}-(1+\gamma) \omega(x) x^{2}+\gamma \omega(x)^{2} x=0
$$

Es bien sabido que para cualquier idempotente e de peso 1. podemos descomponer $\mathbf{A}$ en la forma $\mathbf{A}=K \mathbf{e} \oplus N$ donde $N=\operatorname{ker} \omega \quad$ Sea ahora $x=\mathbf{e}+\lambda n$ con $\lambda \in K$ y $n \in N$ entonces de (1 1) obtenemos las siguientes dos ıdentidades válidas en $N$

$$
\begin{gathered}
\gamma n+2 \mathbf{e}(\mathbf{e} n)-(1+2 \gamma) \mathbf{e} n=0 \\
e n^{2}+2 n(e n)=(1+\gamma) n^{2}
\end{gathered}
$$

Reescribiendo (1.2) obtenemos e $(n-2 \mathbf{e} n)=\gamma(n-2 \mathbf{e} n)$ y $\mathbf{e}(\mathbf{e} n-\gamma n)=$ $\frac{1}{2}($ en $-\gamma n) \quad$ Sea $\tau N \rightarrow N$ el $K$-homomorfismo dado por la multiplicación a la izquierda por el idempotente e. Denotando $U=\left\{n \in N /\right.$ en $\left.=\frac{1}{2} n\right\}$ y $V=\{n \in N / \mathrm{e} n=\gamma n\}$. las igualdades anteriores indican que en $-\gamma n \in$ $\operatorname{ker}\left(\tau-\frac{1}{2} I\right)=U^{I}$ y $n-2$ en $\in \operatorname{ker}(\tau-\gamma I)=V \quad$ Así tenemos que

$$
\left(\frac{1}{2}-\gamma\right) n=(e n-\gamma n)+\frac{1}{2}(n-2 e n) \in U+V
$$

Si $\gamma \neq \frac{1}{2}, N$ se descompone en dos sumandos directos y en consecuencia

$$
\mathbf{A}=K \mathbf{e} \oplus U \oplus V
$$

Linealizando la identidad (1.3) obtenemos las relaciones

$$
U^{2} \subseteq V, \quad U V \subseteq U \quad y \quad V^{2}=0
$$




\section{FORMAS BILINEALES.}

Sea $\mathcal{B}: \mathbf{A} \times \mathbf{A} \rightarrow K$ una forma bilineal, decimos que $\mathcal{B}$ es asociativa, si $\mathcal{B}(a b, c)=\mathcal{B}(a, b c) \forall a, b, c \in \mathbf{A}$ El radical izquierdo de una forma bilineal $\mathcal{B}$ es el conjunto de todos los $x \in \mathbf{A}$ tales que $\mathcal{B}(x, y)=0 \forall y \in \mathbf{A} \quad \mathcal{B}$ es una forma bilineal no degenerada izquierda si su radical izquierdo es cero. Análogamente son definidos los conceptos de radical y forma bilineal no degenerada derechos. Si las formas bilineales fuesen simétricas o antisimétricas $(\mathcal{B}(x, y)=\varepsilon \mathcal{B}(y, x) \forall x, y \in$ A . $\varepsilon= \pm 1$ ) la distinción entre derecha e izquierda desaparece

Consideremos la $\mathcal{T}$-álgebra( $\mathbf{A}, \omega)$ (naturalmente de rango 3 y con un elemento idempotente e) y supongamos $\gamma \neq \frac{1}{2}$ Como $\mathbf{A}=K \mathbf{e} \oplus U \oplus V$ observamos que las hipótesis del Lema que aparece en [1] se satisfacen, pues basta hacer $\lambda_{1}=\frac{1}{2} \quad \lambda_{2}=\gamma \quad N_{1}=U$ y $N_{2}=V \quad$ Utilizando la primera afirmación de este Lema, con $\imath=1$ tenemos $\mathcal{B}(\mathbf{e}, U)=\mathcal{B}(U, \mathbf{e})=0$ Del mismo modo, en la segunda afirmación podemos hacer $i=1, j=2$, obteniendo $\mathcal{B}(U, V)=\mathcal{B}(V, U)=0$ Así la matriz asociada a la forma bilineal $\mathcal{B}$ correspondiente a la descomposición $\mathbf{A}=K \mathbf{e} \oplus U \oplus V$ es

$$
[\mathcal{B}]=\begin{gathered}
\mathbf{e} \\
\mathbf{e} \\
\beta_{1} \\
\beta_{2}
\end{gathered}\left(\begin{array}{ccc}
\mathcal{B}(\mathbf{e}, \mathbf{e}) & 0 & * \\
0 & * & 0 \\
* & 0 & *
\end{array}\right)
$$

donde $\beta_{1}$ es una $K$-base de $U$ y $\beta_{2}$ es una $K$-base de $V$

Proposición 2.1. Si una $T$-álgebrade rango 3 , con idempotente admite una forma bilineal asociativa no degenerada, entonces ella es de Jordan

Demostración: Sea $(\mathbf{A}, \omega)$ una $\mathcal{T}$-álgebracon ecuación rango (1.1) y sea $\mathcal{B}$ una forma bilineal asociativa en $\mathbf{A}$

i) $\operatorname{Si} \gamma=\frac{1}{2}$, tenemos que $n=4 e n-4 e(e n)$, luego para todo $n \in N$ vale la igualdad $\mathcal{B}(\mathrm{e}, n)=\mathcal{B}(\mathrm{e}, 4 \mathrm{en})-\mathcal{B}(\mathbf{e}, 4 \mathrm{e}(\mathrm{e} n))=4\left(\mathcal{B}(\mathrm{e}, \mathrm{e} n)-\mathcal{B}\left(\mathrm{e}^{2}, \mathrm{e} n\right)\right)=0$. es decir $\mathcal{B}(\mathbf{e}, N)=0$ Además para $n_{1}, n_{2} \in N$ arbitrarios, tenemos

$$
\begin{aligned}
\mathcal{B}\left(n_{1}, n_{2}\right) & =\mathcal{B}\left(4 \mathrm{e} n_{1}-4 \mathrm{e}\left(\mathbf{e} n_{1}\right), 4 \mathrm{e} n_{2}-4 \mathbf{e}\left(\mathbf{e} n_{2}\right)\right) \\
& =16\left(\mathcal{B}\left(\mathbf{e} n_{1}, \mathbf{e} n_{2}\right)-\mathcal{B}\left(\mathbf{e} n_{1}, \mathbf{e}\left(\mathbf{e} n_{2}\right)\right)-\mathcal{B}\left(\mathbf{e}\left(\mathbf{e} n_{1}\right), \mathbf{e} n_{2}\right)\right. \\
& +\mathcal{B}\left(\mathbf{e}\left(\mathbf{e} n_{1}\right), \mathbf{e}\left(\mathbf{e} n_{2}\right)\right) \\
& =0
\end{aligned}
$$


puesto que $\mathcal{B}$ es asociativa y $N$ es una subálgebra de $\mathbf{A}$ Luego

$$
[B]=\underset{\beta}{\mathrm{e}}\left(\begin{array}{cc}
\mathrm{e} & \beta \\
\mathcal{B}(\mathbf{e}, \mathbf{e}) & 0 \\
0 & 0
\end{array}\right)
$$

donde $\beta$ es cualquier $K$-base de $N$ Entonces si $\mathcal{B}$ es no degenerada, necesariamente $N=\langle 0\rangle$, de donde $\mathbf{A}=$ Ke y $\mathbf{A}$ es de Jordan

ii) Si $\gamma \neq \frac{1}{2}$, ya hemos visto que la forma canónica matricial en bloques de $\mathcal{B}$ está dada por $(2.1)$ la forma

Cada vez que $0 \neq \gamma \neq 1$, por Lema enunciado en [1]. esta matriz toma

$$
[\mathcal{B}]=\begin{gathered}
\mathrm{e} \\
\beta_{1} \\
\beta_{2}
\end{gathered}\left(\begin{array}{ccc}
\mathcal{B}(\mathrm{e}, \mathrm{e}) & 0 & 0 \\
0 & 0 & 0 \\
0 & 0 & 0
\end{array}\right)
$$

es decir la forma bilineal $\mathcal{B}$ es degenerada, salvo que $N=\langle 0\rangle$ En tal caso tenemos que $\mathbf{A}$ es una $K$-álgebra de Jordan deseado

Cuando $\gamma=0$ o $\gamma=1$. aplicando la Proposición 11. se concluye lo

Proposición 2.2. La forma bilineal asociativa $\mathcal{B}$ definida en una $\mathcal{T}$-álgebrade rango 3 con idempotente es simétrica si y sólo si $\left.\mathcal{B}\right|_{V}$ es simétrica

Demostración: En el caso $\gamma \neq \frac{1}{2}$. observemos que si $v \in V, u_{1}, u_{2} \in U$ entonces valen las igualdades

$$
\begin{gathered}
\mathcal{B}(\mathbf{e}, v)=\mathcal{B}\left(\mathbf{e}^{2}, v\right)=\mathcal{B}(\mathbf{e}, \mathbf{e} v)=\mathcal{B}(\mathbf{e} v, \mathbf{e})=\mathcal{B}\left(v, \mathbf{e}^{2}\right)=\mathcal{B}(v, \mathbf{e}) \mathrm{y} \\
\mathcal{B}\left(u_{1}, u_{2}\right)=2 \mathcal{B}\left(\frac{1}{2} u_{1}, u_{2}\right)=2 \mathcal{B}\left(\mathbf{e} u_{1}, u_{2}\right)=2 \mathcal{B}\left(\mathbf{e}, u_{1} u_{2}\right) \\
=2 \mathcal{B}\left(\mathbf{e} u_{2}, u_{1}\right)=\mathcal{B}\left(u_{2}, u_{1}\right)
\end{gathered}
$$

Luego la simetría de $\mathcal{B}$ sólo depende de la simetría de $\left.\mathcal{B}\right|_{V}$

Si $\gamma=\frac{1}{2}$ se tiene que $\mathcal{B}(x, y) \neq 0$ sólo cuando $x=y=\mathbf{e}$,como se demostró en la Proposición 2.1

Proposición 2.3. Sea $(\mathbf{A}, \omega)$ una $\mathcal{T}$-álgebracon idempotente e tal que $\mathbf{A} \neq K \mathbf{e}$ y cuya ecuación rango está dada por $x^{3}-\omega(x) x^{2}=0$ Entonces las formas bilineales 
asociativas no degeneradas están dadas por las formas bilineales no degeneradas sobre un subespacio vectorial complementario de $U^{2}$ en $V$

Demostración: A es una $\mathcal{T}$-álgebrade rango 3 , con $\gamma=0$ De acuerdo con el teorema demostrado por Walcher, [4], A es simultáneamente de Bernstein y Jordan. En este caso las formas bilineales asociativas no degeneradas están dadas por las formas bilineales no degeneradas sobre un subespacio vectorial complementario de $U^{2}$ en $V$ como se demuestra en [1]

Proposición 2.4. Sea $(\mathbf{A}, \omega)$ una $\mathcal{T}$-álgebracon idempotente e tal que $\mathbf{A} \neq K$ e y cuya ecuación rango sea $x^{3}-2 \omega(x) x^{2}+\omega(x)^{2} x=0$ Entonces cualquier forma bilineal asociativa está determinada por sus valores en $(\mathbf{e}, \mathbf{e})$ y $(\mathbf{e}, V)$

Demostración: A es una $\mathcal{T}$-álgebracon $\gamma=1$ Sea $\mathcal{B}$ una forma bilineal asociativa en $\mathbf{A}$, entonces la forma de su matriz conónica por bloques está dada por (2.1). Cuando $v_{1}, v_{2} \in V$ usamos el hecho que $v_{1} v_{2} \in V^{2}=0$, para probar que $\mathcal{B}\left(v_{1}, v_{2}\right)=0$, es decir que $\mathcal{B}(V . V)=0$ Además si $u_{1}, u_{2} \in U$, notemos que $\mathcal{B}\left(u_{1}, u_{2}\right)=2 \mathcal{B}\left(\frac{1}{2} u_{1}, u_{2}\right)=2 \mathcal{B}\left(\mathrm{e}, u_{1} u_{2}\right)$, luego conocidos los valores que toma $\mathcal{B}(\mathrm{e}, V)$, son conocidos los valores de $\mathcal{B}(U, U)$

Proposición 2.5. Sea $\mathcal{B}$ una forma bilineal asociativa definida en una $\mathcal{T}$-álgebrade rango 3. Si e, $\mathbf{e}_{1}$ son dos idempotentes de peso 1 entonces $\mathcal{B}\left(\mathbf{e}_{1}, \mathbf{e}_{1}\right)=\mathcal{B}(\mathbf{e}, \mathbf{e})$

Demostración: Sea $(\mathbf{A}, \omega)$ la $\mathcal{T}$-álgebra.

i) Supongamos que $\gamma=\frac{1}{2}$ Como $\mathbf{A}=K \mathbf{e} \oplus N$ con $N=\operatorname{ker} \omega$ y $\mathbf{e}_{1}$ es de peso 1, podemos escribir $\mathrm{e}_{1}=\mathbf{e}+n_{0}$ obteniendo $n_{0}=2 \mathrm{e} n_{0}+n_{0}^{2} \mathrm{y}$ $\mathcal{B}\left(n_{0}, n_{0}\right)=4 \mathcal{B}\left(\mathrm{e} n_{0}, \mathrm{e} n_{0}\right)=4 \mathcal{B}\left(\mathrm{e}, n_{0}\left(\mathrm{e} n_{0}\right)\right) \quad$ La identidad (1.2) indica que para cualquier $n \in N$ tenemos $n=4 \mathbf{e} n-4 \mathbf{e}(\mathbf{e} n)$, entonces $\mathcal{B}(\mathbf{e}, n)=0, \mathrm{y}$ en consecuencia $\mathcal{B}\left(n_{0}, n_{0}\right)=0$ Ahora, un cálculo directo prueba la tesis.

ii) Sea $\gamma \neq \frac{1}{2} \quad$ R. Costa demostró en [2], que en este caso existe $u \in U$ tal que $\mathbf{e}_{1}=\mathbf{e}+u+(1-2 \gamma)^{-1} u^{2} \quad$ Es fácil verificar que $B\left(\mathbf{e}_{1}, \mathbf{e}_{1}\right)=$ $\mathcal{B}(\mathbf{e}, \mathbf{e})+\frac{4(1-\gamma)}{(1-2 \gamma)} \mathcal{B}\left(\mathbf{e}, u^{2}\right) \quad$ Observe que sólo debemos preocuparnos del caso $\gamma \neq 1$ Como $\mathcal{B}\left(\mathrm{e}, u^{2}\right)=\mathcal{B}\left(\mathbf{e}, \mathbf{e} u^{2}\right)=\gamma \mathcal{B}\left(\mathbf{e}, u^{2}\right)$, entonces $\mathcal{B}\left(\mathbf{e}, u^{2}\right)=0$

Observación: Bajo las hipótesis de la Proposición 2.5, cada vez que exista $u \in U$ tal que $u^{2}=0$, toda forma bilineal asociativa en $\mathbf{A}$ es degenerada.

\section{REFERENCES}

[1] Baeza R.,Catalán A.,Costa R.: Bernstein algebras with associative bilinear forms.Arch. Math.(por aparecer). 
[2] Costa $R$. Principal train algebras of rank 3 and dimension $\leq 5$. Proccedings of the Edinburg Math. Soc., 33, pp 61-70, 1990.

[3] Ouattara M. Algebres de Jordan et algebres genetiques. Tesis Doctorado, Montpellier. Francia, 1989.

[4] Walcher S. Bernstein algebras which are Jordan algebras. Arch. Math. 50, pp 218-222. 1988.

Recibido: 9 de Septıembre de 1991

R. Baeza-Vega y R. Benavides

Departamento de Matemáticas y Estadística

Universidad de la Frontera

Casilla 54-D. Temuco. Chile 